\title{
Exploring and exploiting the antibody and Ig superfamily combining sites
}

\author{
Andrew George, James S. Huston, and Edgar Haber
}

As a result of recent progress in molecular immunology and antibody engineering, we are in a very exciting phase in the exploitation of antibody and immunoglobulin (Ig) superfamily binding regions. The progress has come about because of the increase in our understanding of Ig superfamily interactions and structure, the ease with which the molecules can now be genetically modified, a fuller understanding of Ig genetics, and the ability to select recombinant binding regions using phage-display technology. At a recent conference ${ }^{1}$, these strands were brought together.

Fundamental to the exploitation of antibodies is a full understanding of Ig genetics. One of the most exciting features of the conference was how sequence data are being related to combining-site structure and maturation during the immune response (Ian Tomlinson, Cambridge, UK). The Ig heavy chain and $\operatorname{Ig}(\kappa$ and $\lambda$ ) light chain loci have been mapped in human genomic DNA, and the $\mathrm{V}, \mathrm{D}$, and $\mathrm{J}$ segments have been sequenced. Somewhat surprisingly, there is less diversity in the number of functional $\mathrm{V}$ regions than was previously expected, with only 51 heavy chain V, $40 \mathrm{VK}$ and approximately $30 \mathrm{~V} \lambda$ segments.

Knowledge of the sequences has allowed identification of the germline sequence that corresponds to any human Ig sequence. The residues that differentiate germline sequences, as well as those that have been altered in the course of somatic hypermutation can, therefore, be identified, and their position indicated on a three-dimensional model of the antigen combining site.

Interestingly, the majority of the diversity caused by differences in the germline sequences is concentrated at the center of the antigen-binding site. During somatic mutation, diversity spreads to the periphery of the site, suggesting that the two mechanisms of diversity are complementary and may have coevolved to provide an efficient way of

Andrew George is at the department of immunology, Royal Postgraduate Medical School, Hammersmith Hospital, London WI2 oNN,UK (ageorge@rpms.ac.uk); James S. Huston is at Creative Biomolecules, 4 South Street, Hopkinton, MA 01748; Edgar Haber is at the Center for the Prevention of Cardiovascular Disease, Harvard School of Public Health, 677 Huntington Avenue, Boston, MA 02115. searching sequence space for the best antigen-binding molecule.

Molecular immunology also provides powerful tools. Probably the most commonly used recombinant antibody fragment is the single-chain $\mathrm{Fv}(\mathrm{sFv})$, which consists of the heavy and light chain variable regions joined by a linker peptide. Phage display methods allow researchers to produce either novel specificities or improved affinities ${ }^{2,3}$.

\section{One interesting application of the intracellular expression of recombinant antibodies in therapy is to modify the phenotype of cells.}

One interesting application of the intracellular expression of recombinant antibodies in therapy is to modify the phenotype of cells (Wayne Marasco, Boston, MA). Recombinant antibody fragments can be fused to a variety of intracellular trafficking signals that direct the antibody to different cellular locations. For example, production of $\mathrm{sFv}$ specific for a cell-membrane protein, fused with endoplasmic-reticulum retention signals, will cause the protein to be held in the endoplasmic reticulum, where it will eventually be degraded and not reach the cell surface. In addition, intracellular antibodies have been generated against all 12 viral proteins expressed within cells during the course of HIV infection; some of the intracellular antibodies have been shown to inhibit viral replication or infection, opening the prospect for "intracellular immunization" against viral infection.

The extension of molecular immunology into the realm of the Ig superfamily was another major focus of the meeting. Significant new insights were described on the nature of the interaction between the T-cell antigen receptor (TCR) and its antigen. In particular, the first crystal structure of the TCR $\beta$ chain complexed with a superantigen, staphylococcal enterotoxin C3 (SEC3), was described (Roy Mariuzza, Rockville, MD). The SEC3 binds exclusively to the CDR1, CDR2, and the fourth hypervariable loop of the $V \beta$ domain, its orientation being such that it would act as a wedge between the TCR and the peptide-MHC complex, preventing peptide-TCR interactions.

In conventional antigen recognition, a trimolecular complex is formed, involving the MHC, peptide, and TCR. The role of the peptide in the complex is complicated, with some residues interacting with pockets in the MHC groove, and others making direct contact with the TCR. It has been shown that this can cause cross-recognition; for example, peptides derived from common viruses can bind the MHC-DR2 molecules and be recognized by $T$ cells specific for myelin basic protein (Jack Strominger, Cambridge, MA). The concept of degenerate molecular mimicry may be important in the etiology of a range of autoimmune diseases.

Much of the work presented at the meeting was in the preclinical stage. It was, therefore, encouraging to see data concerning the use of novel recombinant molecules in patients. Phage-display technology has been used to generate an sFv directed against carcinoembryonic antigen. The molecule, labeled with ${ }^{123} \mathrm{I}$, has been used to image patients with colorectal or breast carcinoma (Richard Begent, London).

One of the potential advantages of using such reagents as $\mathrm{sFv}$ is that their small size ensures rapid clearance from the circulation. In trial patients the $\mathrm{sFv}$ did indeed clear rapidly, with biphasic kinetics $(\mathrm{t} 1 / 2 \mathrm{a}=0.4 \mathrm{hrs}$, $\mathrm{t} 1 / 2 \mathrm{~b}=5.3 \mathrm{hrs}$ ), resulting in good localization of the sFv within one hour of injection. This allows rapid and sensitive detection of tumor deposits in patients. In addition, an immunotoxin, comprising a truncated version of Pseudomonas exotoxin with a sFv with specificity for the Ley carbohydrate epitope expressed on a range of tumors, is undergoing initial toxicity testing (Ira Pastan, Bethesda, MD). The dose escalation schedule has just reached the levels where animal models suggest a therapeutic effect may start to be seen, with no significant toxicity. It is likely that we will see similar trials of other agents that use the Fv for targeting in the future.

\footnotetext{
1. February 22-28, 1996, Taos, NM; presented by the Keystone Symposia on Molecular and Cellular Biology. The abstracts for the meeting will be published in Immunotechnology.

2. Winter, G., Griffiths, A.D., Hawkins, R.E., and Hoogenboom, H.R. 1994. Annu. Rev. Immunol. 12:433-455.

3. Chester, K.A. and Hawkins, R.E. 1995. Trends Biotechnol. 13:294-300.
} 